\title{
Levitation Stability of the Passive Magnetic Bearing in a Nutation Blood Pump
}

\author{
Gang Chen, ${ }^{1,2}$ Li-Gang Yao, ${ }^{1}$ Kun-Chieh Wang, ${ }^{2 *}$ Jia-Xin Ding, ${ }^{1}$ and Zhen-Ya Wang ${ }^{1}$ \\ ${ }^{1}$ School of Mechanical Engineering and Automation, Fuzhou University, Fuzhou 350116, China \\ ${ }^{2}$ School of Mechanical and Electric Engineering, Sanming University, Sanming 365004, Fujian Province, China
}

(Received March 23, 2021; accepted June 22, 2021)

Keywords: nutation blood pump, passive magnetic bearing, levitation stability, analytical model, realtime test

Rotor stability is an important index of the performance of a magnetically levitated blood pump. In this study, we developed a novel nutation blood pump using a passive magnetic spherical bearing to achieve dynamic stability of a levitated rotor. The structure and working principle of the proposed blood pump are first introduced. A mathematical model based on the theory of equivalent magnetic charges is derived to calculate the axial and rotational stiffnesses of the passive magnetic spherical bearing. Furthermore, considering the gyro effect on the dynamic stability, an analytical expression for the gyroscopic moment of the nutating rotor is deduced. Finally, a prototype of a self-designed blood pump is fabricated, and a hydraulic experiment on the real-time levitation state is carried out. Experimental results obtained via different sensing components show that a continuous output flow rate of $5 \mathrm{~L} / \mathrm{min}$ can be obtained against a pressure head of $100 \mathrm{mmHg}$ at a rotational speed of $1600 \mathrm{rpm}$, and the output flow conditions are found to be stable at various pressures. In addition, it is found that the rotor becomes more stable with increasing rotational speed. The maximum fluctuation of the levitated gap is only $0.2 \mathrm{~mm}$ when the rotational speed of the rotor is $2300 \mathrm{rpm}$.

\section{Introduction}

The blood pump has been widely studied as an important component of the total artificial heart (TAH) and the ventricular assist device (VAD). The magnetically levitated blood pump, as the third-generation artificial heart pump without a mechanical bearing that has high durability and low hemolytic properties as well as anti-thrombogenicity, is a leading candidate for TAHs and VADs. ${ }^{(1,2)}$ The passive magnetic bearing, which does not have any additional energy consumption or complex control systems, is regarded as the most promising magnetic bearing for use in blood pumps. (3) Several blood pumps with passive magnetic bearings have been developed for use in clinical settings, such as the Valvo pump, ${ }^{(4)}$ HeartWare HVAD, ${ }^{(5)}$ and Arrow CorAide. ${ }^{(6)}$ Furthermore, Chen et al. ${ }^{(7)}$ proposed a passive magnetically levitated nutation blood pump with advantages of a small volume rate and low rotational speed. They

*Corresponding author: e-mail: 20190207@fjsmu.edu.cn https://doi.org/10.18494/SAM.2021.3395 
experimentally verified the structure and principle of blood flow in the pump. However, they did not study the levitation stability of the passive magnetic bearing.

Previous studies have reported many issues concerning the levitation stability of magnetic bearings in various fields. Soni et al. ${ }^{(8)}$ analyzed the stability of an active magnetic bearing (AMB) under parametric excitation. The stability analysis was carried out on the basis of the Floquet-Liapunov theory for the stability of a periodic system. Jarroux et al. ${ }^{(9)}$ assessed the stability of a rotating machine supported on AMBs during severe foundation excitation. The combined effects of unbalanced forces, base motion excitation, and contact nonlinearity on a rotor-AMB system response were analyzed, focusing on the capacity of an augmented proportional-integral-derivative controller to maintain the stability of the system. Yu et al. ${ }^{(10)}$ studied the stability and performance of an AMB flywheel system. To achieve high stability at ultrahigh flywheel speeds with low power consumption, cross-feedback PID control was applied to the AMB flywheel system. In this experimental study, the gyroscopic effect of the flywheel was effectively suppressed. Note that all the above studies were targeted at the stability of an AMB.

Zhang et al. ${ }^{(11)}$ carried out a theoretical analysis of a permanent magnetic bearing system with six magnetic rings and five degrees of freedom, and found that it was possible to achieve dynamic stability in the passive full permanent magnet bearing system. Xia et al. ${ }^{(12)}$ analyzed the stability of a rotor system in a magnetically suspended control and a sensitive gyroscope with the rotation of a Lorentz force magnetic bearing. They established a dynamic model for this system that considers the rotation of the Lorentz force magnetic bearing, and proposed a stability analysis method based on a pseudolinear equivalent transformation and extended the doublefrequency Bode diagram. Ambwani et al. ${ }^{(13)}$ presented a novel axial vibration suppression method in two-axis actively positioned permanent magnet bearingless motors. Shida et al. ${ }^{(14)}$ studied the radial stability of a magnetically levitated impeller in a centrifugal blood pump with an axially symmetric magnetic suspension system and found that widening the radial clearance is an effective approach to improve the radial stability as well as hemocompatibility. To measure the levitated gap, Bente et al. ${ }^{(15)}$ studied a method that uses X-ray radiographic imaging to measure the axial gap clearance of a radial rotary blood pump with a combination of passive magnetic and hydrodynamic bearings.

Despite the aforementioned studies, which mainly focused on a rotor around a fixed axis, the levitation stability of a nutating rotor has not yet been fully investigated. In this study, an analysis of the levitation stability of a passive magnetic bearing in the nutation process is presented. First, a novel topology of a passive magnetic nutation blood pump is introduced. Second, the possibility of dynamic stability of the nutating rotor under the gyroscopic effect is analyzed in Sect. 2. Then, real-time monitoring of the rotor at various rotational speeds is reported in Sects. 3 and 4.

\section{Analysis of Levitation Stability}

To improve the levitation stability of the magnetic bearing in a nutation blood pump, we have designed a novel magnetically levitated structure. On the basis of the following theoretical 
analysis, which adopts the theory of equivalent magnetic charges and the gyroscopic effect, we obtain high levitation stability for our proposed passive magnetic bearing.

\subsection{Structure and principle of passive magnetic nutation blood pump}

Figure 1 shows our newly designed layout of a passive magnetic blood pump. We use six pairs of permanent magnets with fan-shaped magnetic sleeves, which are orthogonally arranged in the bearing. Past arrangements of permanent magnets usually had a defect of large magneticforce fluctuation of the bearing during rotation. ${ }^{(7)}$ As shown in Fig. 1, there are only two pairs of magnetic rings in the bearing of the current blood pump. For the first pair, one magnetic ring is set in the lower shell of the pump and the other is set in the lower spherical rotor to generate the expected repulsive force. For the second pair, one repulsive magnetic ring is set in the upper shell and the other is set in the upper spherical rotor. The repulsion force between these two pairs of magnetic rings keeps the rotor levitated to reduce mechanical wear and thus prevent blood from being damaged. In addition, seven permanent magnets with the same magnetic poles are uniformly embedded in the edge of the nutation disk. Two permanent magnets with opposite poles are fixed on the motor rotor in a straight line. One of the magnets is attracted to the magnets on the edge of the nutation disk and the other repels it, which together keep the nutation disk tilted and in contact with the inner cones of the upper and lower shells in lines. The contact lines rotate with the motor rotor and the nutation disk swings up and down in the direction of these contact lines. When the rotor of the pump nutates, the inlet area increases and thus a negative-pressure condition will form, which causes the fluid to continuously flow into the pump room. At the same time, the outlet area decreases, the fluid is pushed out through the outlet, and the pump mechanism is finally activated.

\subsection{Calculation of magnetic force and torque}

From the theory of equivalent magnetic charges, the interaction force between magnetic charges can be illustrated by the Coulomb law between electric charges. ${ }^{(16)}$ Considering two
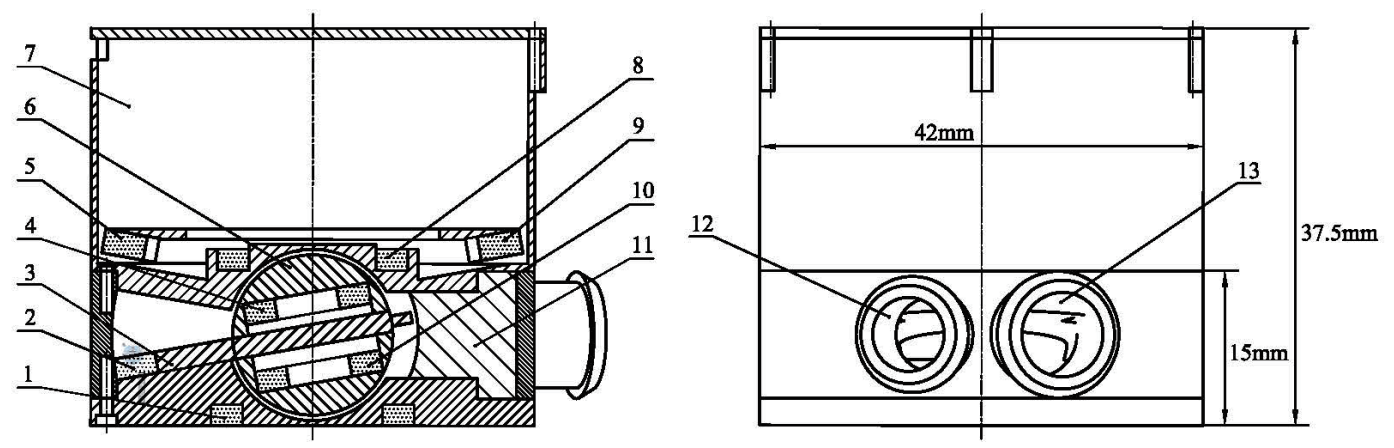

Fig. 1. Layout of the magnetic nutation blood pump. 1: Magnetic ring in the lower shell. 2: Magnet in the nutation disk. 3: Nutation disk. 4: Upper magnetic ring in the rotor. 5: Drive magnet (S). 6: Rotor of the nutation pump. 7: Motor. 8: Magnetic ring in the upper shell. 9: Drive magnet $(\mathrm{N})$. 10: Lower magnetic ring in the rotor. 11: Baffle plate. 12: Outlet. 13: Inlet. 
point magnetic charges 1 and 2 , if the magnetic intensity and the distance between the magnetic charges are known and magnetic charge 2 is in the magnetic field of magnetic charge 1 , the interaction force between them can be expressed as

$$
\boldsymbol{F}_{12}=\frac{1}{4 \pi \mu_{0}} \cdot \frac{\boldsymbol{q}_{1} \boldsymbol{q}_{2}}{\left|\boldsymbol{r}_{12}\right|^{3}} \cdot \boldsymbol{r}_{12}
$$

where $\boldsymbol{q}_{1}$ and $\boldsymbol{q}_{2}$ are the magnetic charges of points 1 and 2, respectively, $\left|\boldsymbol{r}_{12}\right|$ is the distance between point magnetic charges 1 and $2, \mu_{0}$ is the permeability of vacuum, and $\mu_{0}=$ $4 \pi \times 10^{-7} \mathrm{H} \cdot \mathrm{m}^{-1}$.

The two magnetic rings $S_{\mathrm{I}}$ and $S_{\mathrm{II}}$ are both made of Nb-Fe-B permanent magnet and have dimensions $R_{1}, R_{2}$, and $2 L_{1}$ and $R_{3}, R_{4}$, and $2 L_{2}$, respectively, as shown in Fig. 2 . The magnetic rings are magnetized along their axial directions and their lengths are equal, i.e., $L_{1}=L_{2}$. The magnetic charge densities on the end surfaces of the rings are $\sigma_{1}$ and $\sigma_{2}$, which are assumed to be sufficiently high and uniform. $S_{\mathrm{I}}$ is fixed in the pump shell and $S_{\mathrm{II}}$ is in the center of the nutation disk. $S_{\mathrm{I}}$ and $S_{\mathrm{II}}$ are a distance of $e$ apart along the $z_{0}$ axis. $z_{1}$ is the axis of $S_{\mathrm{I}} . O_{0}$ and $O_{1}$ are the centers of $S_{\text {I }}$ and $S_{\text {II }}$, respectively. $S_{\text {II }}$ undergoes nutation together with the nutation disk, but no self-rotation. Thus, the $x_{1}$ and $y_{1}$ axes swing up and down in the $x_{0} o_{0} z_{0}$ and $y_{0} o_{0} z_{0}$ planes, respectively. The angles between $x_{1}$ and $x_{0}$ and between $y_{1}$ and $y_{0}$ change with time but vary in the range from $-\theta$ to $\theta$, where $\theta$ is the nutation angle.

The interaction forces along the $x_{0}, y_{0}$, and $z_{0}$ axes are the sum of four forces, where two forces are located between end surfaces 1 and 2 of $S_{\mathrm{I}}$ and the other two forces are located on end surfaces 3 and 4 of $S_{\text {II }}$, as listed below:

$$
\left.\begin{array}{l}
\boldsymbol{F}_{x}=-\boldsymbol{F}_{13 x}+\boldsymbol{F}_{23 x}+\boldsymbol{F}_{14 x}-\boldsymbol{F}_{24 x} \\
\boldsymbol{F}_{y}=-\boldsymbol{F}_{13 y}+\boldsymbol{F}_{23 y}+\boldsymbol{F}_{14 y}-\boldsymbol{F}_{24 y} \\
\boldsymbol{F}_{z}=-\boldsymbol{F}_{13 z}+\boldsymbol{F}_{23 z}+\boldsymbol{F}_{14 z}-\boldsymbol{F}_{24 z}
\end{array}\right\} .
$$

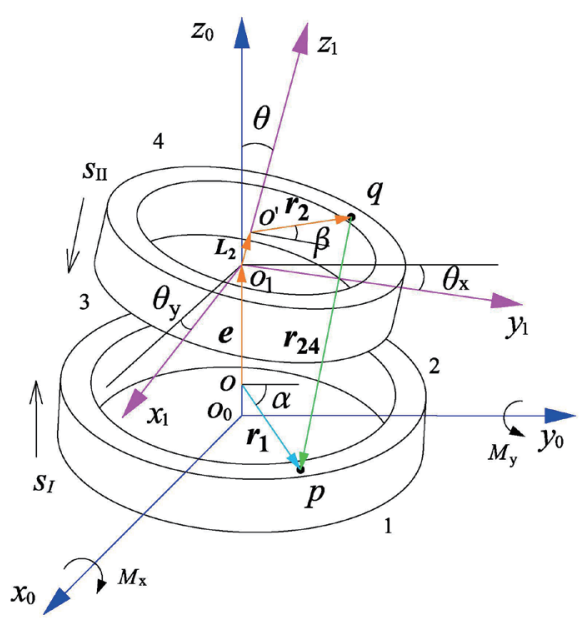

Fig. 2. (Color online) Relative positions of the magnetic rings in the nutation process. 
In this case, we assume without loss of generality that the attractive force is negative while the repulsive force is positive. The integrative form of the force can be expressed as

$$
\left.\begin{array}{l}
F_{x}=\frac{B_{\mathrm{r} 1} B_{\mathrm{r} 2}}{4 \pi \mu_{0}} \int_{0}^{2 \pi} \int_{0}^{2 \pi} \int_{R_{1}}^{R_{2}} \int_{R_{3}}^{R_{4}}\left(-\frac{r_{13 x}}{\left|r_{13}\right|^{3}}+\frac{r_{23 x}}{\left|r_{23}\right|^{3}}+\frac{r_{14 x}}{\left|r_{14}\right|^{3}}-\frac{r_{24 x}}{\left|r_{24}\right|^{3}}\right) r_{1} r_{2} d r_{1} d r_{2} d \alpha d \beta \\
F_{y}=\frac{B_{\mathrm{r} 1} B_{\mathrm{r} 2}}{4 \pi \mu_{0}} \int_{0}^{2 \pi} \int_{0}^{2 \pi} \int_{0}^{R_{2}} \int_{R_{1}}^{R_{4}}\left(-\frac{r_{13 y}}{\left|r_{13}\right|^{3}}+\frac{r_{23 y}}{\left|r_{23}\right|^{3}}+\frac{r_{14 y}}{\left|r_{14}\right|^{3}}-\frac{r_{24 y}}{\left|r_{24}\right|^{3}}\right) r_{1} r_{2} d r_{1} d r_{2} d \alpha d \beta \\
F_{z}=\frac{B_{\mathrm{r} 1} B_{\mathrm{r} 2}}{4 \pi \mu_{0}} \int_{0}^{2 \pi} \int_{0}^{2 \pi} \int_{R_{1}}^{R_{2}} \int_{R_{3}}^{R_{4}}\left(-\frac{r_{13 z}}{\left|r_{13}\right|^{3}}+\frac{r_{23 z}}{\left|r_{23}\right|^{3}}+\frac{r_{14 z}}{\left|r_{14}\right|^{3}}-\frac{r_{24 z}}{\left|r_{24}\right|^{3}}\right) r_{1} r_{2} d r_{1} d r_{2} d \alpha d \beta
\end{array}\right\}
$$

where $B_{r 1}$ and $B_{r 2}$ are the remanences of magnetic rings $S_{\mathrm{I}}$ and $S_{\mathrm{II}}$, respectively, $r_{1}$ is the distance from any point on surface 1 to its center, $r_{2}$ is the distance from any point on surface 3 to its center, $\alpha$ is the direction angle of vector $\boldsymbol{r}_{1}$ on surface 1 , and $\beta$ is the direction angle of vector $\boldsymbol{r}_{2}$ on surface 3. Since surfaces 1 and 2 have the same geometric shape, the integration variables for surface 2 can be substituted by the variables for surface $1\left(r_{1}, \alpha\right)$. Similarly, the integration variables for surface 4 can be substituted by the variables for surface $3\left(r_{2}, \beta\right)$.

The external driving torque keeps the nutation disk tilted and in contact with the inner cones of the upper and lower shells in line, and keeps the magnetic ring $S_{\text {II }}$ in the nutation disk always tilted. The analytical expression for the magnetic torque between $S_{\mathrm{I}}$ and $S_{\mathrm{II}}$ is established when $S_{\text {II }}$ tilts in a general position (as shown in Fig. 2). It is assumed that the counterclockwise torque is positive and the clockwise torque is negative. The magnetic torque of $S_{\mathrm{II}}$ around the $x_{0}$ axis is

$$
M_{x}=-e F_{y},
$$

where $e$ is the offset distance between $S_{\mathrm{II}}$ and $S_{\mathrm{I}}$ along the $z_{0}$ axis.

Since there is no radial eccentricity for the magnetic bearing, the moment arms of forces $F_{x}$ and $F_{y}$ with respect to the $z_{0}$ axis are zero. Thus, the magnetic bearing has no magnetic torque around the $z_{0}$ axis and also no radial bearing capacity. The linear relationship between the radial bearing capacity of a permanent magnetic bearing and the radial eccentricity is consistent with the study of Li et al. ${ }^{(17)}$

\subsection{Mechanical properties of permanent magnetic bearing in static state}

A stable levitation state of the permanent magnetic bearing in a pump chamber means that both the radial and axial stabilities of levitation are achieved. According to the previous analysis of the magnetic torque, the magnetic bearing cannot attain stability in the radial direction. According to Earnshaw's theorem, a permanent maglev cannot achieve stable equilibrium under a static state in which only the permanent magnetic force acts. However, in our novel design, the radial movement of the nutation disk is restricted by a baffle plate that is installed between the 
inlet and outlet to avoid the reflux of blood in the pump chamber. Therefore, the nutation disk maintains a non-contact state with the inner wall of the pump. The gap length in the radial direction between the disk and inner wall is $0.1 \mathrm{~mm}$. In other words, the permanent magnetic bearing may remain stably levitated in the radial direction through our novel design of the pump structure.

Figure 3 shows the axial magnetic forces of the magnetic rings in the nutation disk. Supposing that the magnetic bearing moves by an offset in the $z$ direction under the application of an external load $F_{z}$, then the external load can be expressed as

$$
F_{z}=F_{z 1}+F_{z 2}-F_{z 3}-F_{z 4},
$$

where $F_{z 1}$ is the force of the magnetic ring in the lower shell applied to the lower magnetic ring, $F_{z 2}$ is the force of the magnetic ring in the lower shell applied to the upper magnetic ring, $F_{z 3}$ is the force of the magnetic ring in the upper shell applied to the upper magnetic ring, and $F_{z 4}$ is the force of the magnetic ring in the upper shell applied to the lower magnetic ring.

The torque of the magnetic rings in the nutation disk around the $x$ axis is

$$
M_{x}=M_{x 1}-M_{x 2}+M_{x 3}-M_{x 4}+M_{x 5}
$$

where $M_{x 1}$ is the torque between the magnetic ring in the lower shell and the lower magnetic ring in the nutation disk, $M_{x 2}$ is the torque between the magnetic ring in the lower shell and the upper magnetic ring in the nutation disk, $M_{x 3}$ is the torque between the magnetic ring in the upper shell and the upper magnetic ring in the nutation disk, $M_{x 4}$ is the torque between the magnetic ring in the upper shell and the lower magnetic ring in the nutation disk, and $M_{x 5}$ is the torque between the two magnets in the rotary sleeve and the magnets in the edge of the nutation disk.

Thus, the axial stiffness of the magnetic bearing can be calculated as

$$
K_{z}=\frac{d F_{z}}{d z}
$$

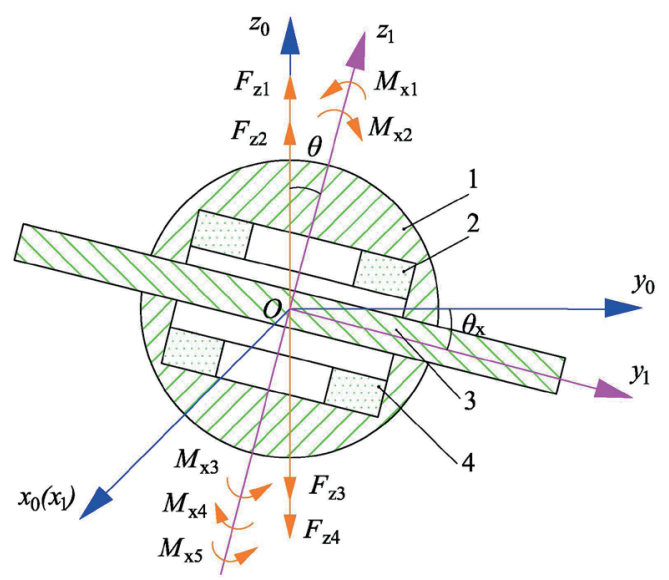

Fig. 3. (Color online) Axial magnetic forces and torques around the $x$ axis of the magnetic rings in the nutation disk: 1: pair of semi-spherical shells, 2: upper magnetic ring, 3: nutation disk, 4: lower magnetic ring. 
The rotational stiffness of the magnetic bearing is obtained as

$$
K_{\theta}=\frac{d M_{x}}{d \theta_{x}}
$$

Through calculations via Eqs. (5) and (7), we may obtain the characteristic curves of the axial bearing capacity with different nutation angles as shown in Fig. 4. Figure 4(a) shows that the axial magnetic force is inversely proportional to the nutation angle when the axial displacement of the magnetic bearing is fixed. The axial magnetic force increases with increasing axial displacement. On the other hand, the result for different axial stiffnesses is shown in Fig. 4(b). The axial stiffness of the bearing is approximately inversely proportional to the axial displacement and is always positive, which indicates that the magnetic bearing can be levitated stably under the action of axial external forces.

However, as shown in Fig. 5(a), the torque of the magnetic bearing is negative and its magnitude is very small, which demonstrates that the magnetic bearing cannot sustain external

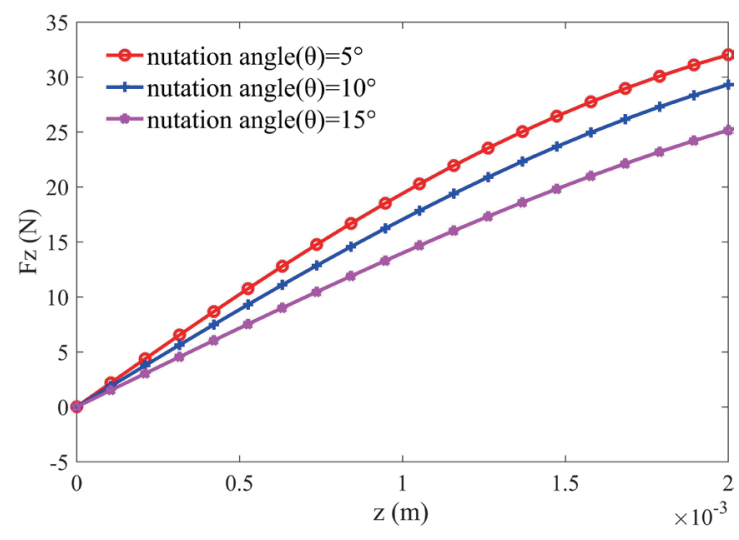

(a)

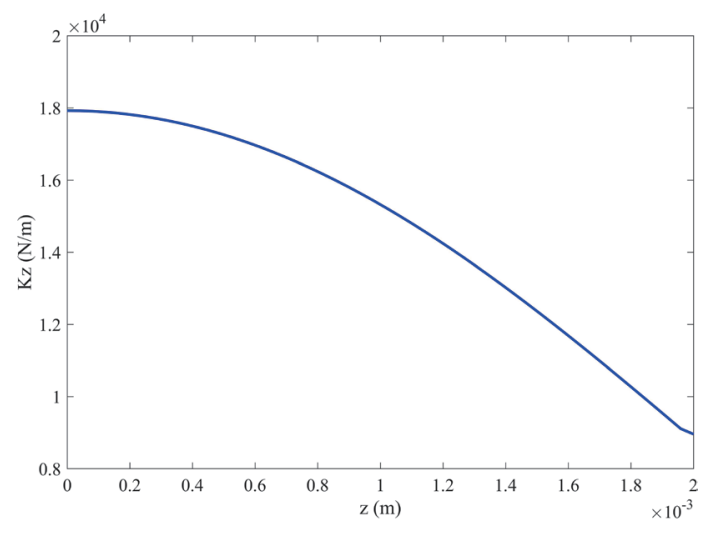

(b)

Fig. 4. (Color online) (a) Relationship between the axial bearing capacity and the axial displacement for different nutation angles. (b) Axial stiffness at the nutation angle of $10^{\circ}$.

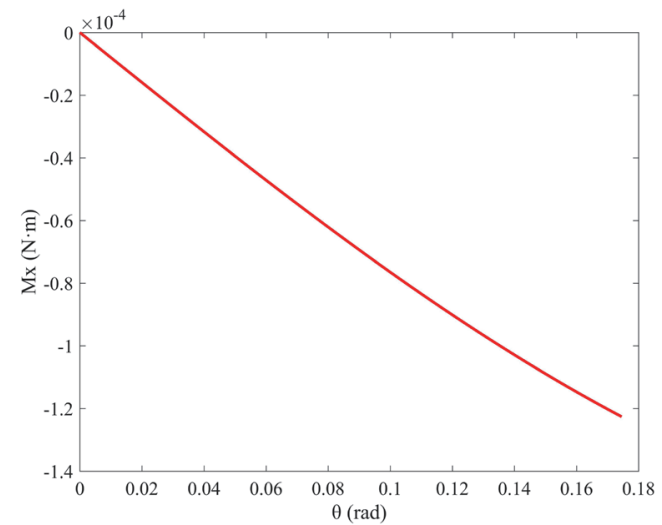

(a)

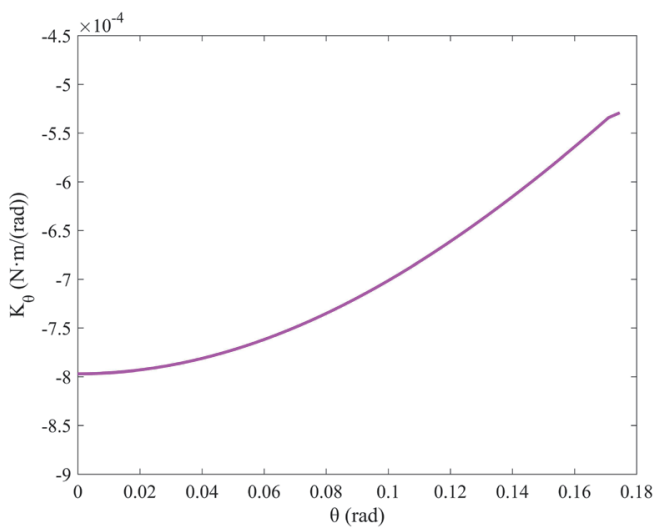

(b)

Fig. 5. (Color online) (a) Variation of the bearing torque with the deflection angle. (b) Variation of the rotational stiffness with the deflection angle. 
torque in the static state. In addition, it can be seen from Fig. 5(b) that the rotational stiffness is also negative. This phenomenon also illustrates that the passive magnetic bearing cannot be levitated stably in the static state under the action of external torques.

\subsection{Analysis of stable levitation of permanent magnetic bearing under dynamic condition}

A permanent magnetic bearing can be levitated stably along the axial direction in the static state, but it is no longer stable when additional torque is applied. This result is consistent with Earnshaw's theorem that a static system consisting of particles that either repel or attract one another is unstable. However, for the well-known Levitron magnetic device, it has been theoretically proven that stability may be achieved once the components of a magnetic bearing or system are in proper relative motion. ${ }^{(18,19)}$ As shown in Fig. 6(a), the Levitron magnetic device, consisting of a permanent magnet that acts as a spinning top, can float in air around an equilibrium position above a heavy base made of a magnetized ceramic square slab. The stability of the Levitron device is induced from the gyroscopic effect of the rotating magnetic elements. Some mathematical models have been presented to describe this effect of the Levitron passive magnetic device. ${ }^{(20)}$ Similar motion features have been found between the magnetically levitated rotor of the nutation blood pump and the Levitron device.

A diagram of our newly designed levitated rotor is shown in Fig. 6(b). The rotor consists of a nutation disk and a pair of semi-spherical shells with a magnetic ring inside. $O-\xi \eta \zeta$ is a reference frame fixed to the ground and $O-x y z$ is a dynamic coordinate system connected with the rotor. The nutation disk undergoes nutation together with the nutation disk, and the $z$ axis of the rotor rotates around the vertical axis $\zeta$. The attitude of the rotor is determined by Euler angles $\psi, \theta$, and $\varphi$.

The rotor is regarded as a rigid body, and its moment of momentum is

$$
\boldsymbol{H}=\boldsymbol{J} \cdot \boldsymbol{\omega}=\left[\begin{array}{c}
J_{x x} \omega_{x}-J_{x y} \omega_{y}-J_{z x} \omega_{z} \\
-J_{y x} \omega_{x}+J_{y y} \omega_{y}-J_{y z} \omega_{z} \\
-J_{z x} \omega_{x}-J_{y z} \omega_{y}+J_{z z} \omega_{z}
\end{array}\right]=\left(A \omega_{x}, B \omega_{y}, C \omega_{z}\right)^{T},
$$

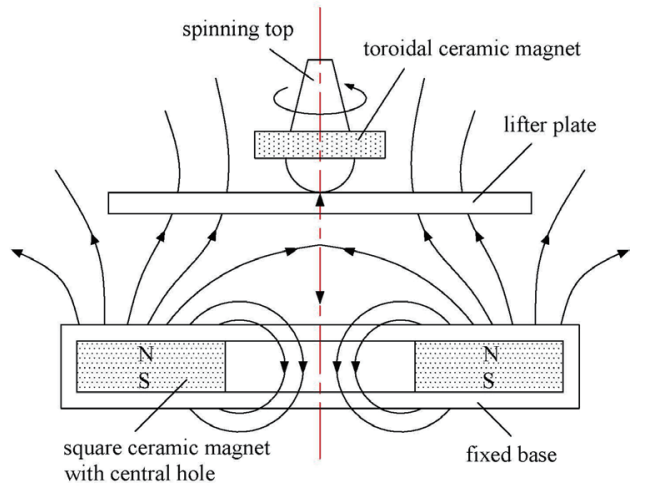

(a)

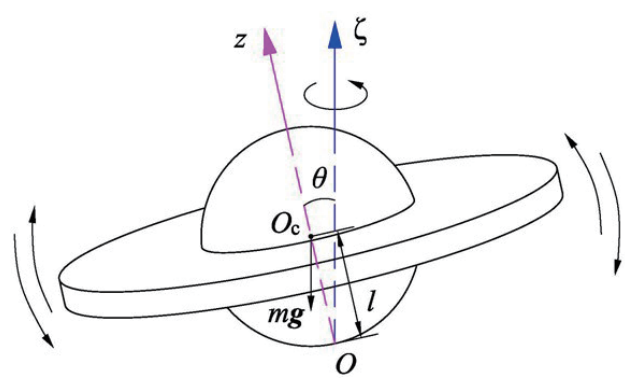

(b)

Fig. 6. (Color online) (a) Sketch of the Levitron device. (b) Motion of the rotor in the nutation blood pump. 
where $\omega$ is the angular speed of the rotor about the contact line between the nutation disk and the upper and lower shells, $\omega_{x}, \omega_{y}$, and $\omega_{z}$ are the components of the angular speed in the $x, y$, and $z$ directions, and $A, B$, and $C$ are the moments of inertia around the $x, y$, and $z$ axes, respectively. They can be expressed as

$$
\left.\begin{array}{l}
A=J_{x}=\sum m\left(y^{2}+z^{2}\right) \\
B=J_{y}=\sum m\left(z^{2}+x^{2}\right) \\
C=J_{z}=\sum m\left(x^{2}+y^{2}\right)
\end{array}\right\}
$$

and

$$
\omega_{x}=\dot{\theta}, \omega_{y}=\dot{\psi} \sin \theta, \omega_{z}=\dot{\psi} \cos \theta+\dot{\varphi} .
$$

When the direction and mode of the momentum moment of the rotor relative to the dynamic coordinate system $O-x y z$ remain unchanged, the external moment is calculated as ${ }^{(21)}$

$$
\boldsymbol{M}_{d}=\frac{d \boldsymbol{H}}{d t}=\boldsymbol{\omega} \times \boldsymbol{H}
$$

If the rotor is levitated stably, the resultant moment received by the rotor is zero. Consequently, the following expression is obtained:

$$
\boldsymbol{M}_{d}+\boldsymbol{M}_{o}=0
$$

where $\boldsymbol{M}_{o}$ is the gyroscopic moment.

According to the research by Zhang et al., ${ }^{(11)}$ under the action of the gyroscopic moment, the rotor can achieve stable levitation when its speed is greater than the critical speed. The critical speed is

$$
n_{c r}=\frac{60}{\pi J_{z}} \sqrt{J_{x}\left(m g l \cos \theta+M_{d}\right)}
$$

In brief, we not only illustrate the possibility of achieving levitation stability for our newly designed magnetic system in the dynamic state but also introduce a formula to acquire the critical speed for stability in that state.

\section{Experimental Measurement and Real-time Test of Levitation State}

A hydraulic experiment on our newly designed pump was performed with mock circulation measurements. To simulate a real blood flow, we used 33\% glycerin solution diluted with saline 
water as the testing fluid, whose viscosity is similar to that of blood. The pump output was measured with an electronic turbine flowmeter sensor (K24, Bangsheng Machinery Co. Ltd., Wenzhou, China) attached at the outflow tube. The pressure head of the pump, which is defined as the pressure difference between the outlet and inlet ports, was measured with differential pressure gauge sensors (GM520, Jumaoyuan Science and Technology Co. Ltd., Shenzhen, China) attached at the outflow and inflow tubes. The preload of the pump was adjusted according to the height of the storage container and the after-load was regulated by a throttle valve.

To verify the analysis results, we built a system for real-time monitoring of the rotor in the levitation state. The frame of the monitoring system is shown in Fig. 7. The rotating speed of the blood pump was measured by a photoelectric switch sensor (6234P, Beicheng Electronic Technology Co. Ltd., Xi'an, China). The axial displacement of the rotor was monitored by a noncontact eddy current sensor (ZH3010, Zhonghang Technology Co. Ltd., Zhuzhou, China). The eddy current displacement sensor has the advantages of strong anti-interference, a large dynamic range of measurement, and high stability. The operation principle of the eddy current sensor is shown in Fig. 8. With this sensor, the magnetic flux on the surface of the conductor may be measured when a high-frequency alternating current passes through the coil. Then the distance between the specimen and the surface of the conductor can be measured by the change in the magnetic flux, and the change in the distance can also be measured.

A real-time test measurement with sensing components was performed to monitor the levitation state of the rotor, and a test platform was built, as shown in Fig. 9. The probe of the eddy current sensor was installed in the central threaded hole of the blood pump. The initial distance of the probe to the monitored surface of the rotor was $0.5 \mathrm{~mm}$. The dynamicdisplacement monitoring system of the rotor was composed of a self-designed prototype pump, a control device, a power supply system, non-contact eddy current sensors, a signal monitor, a

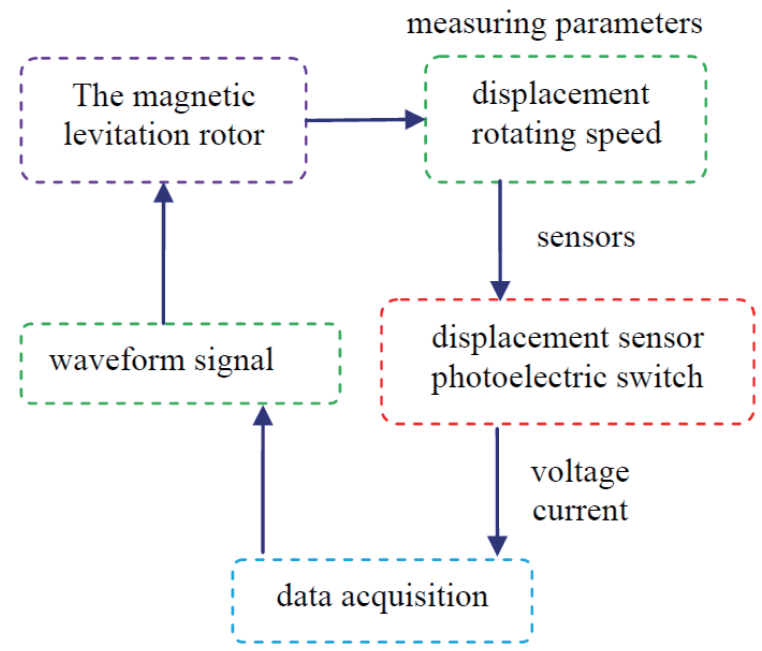

Fig. 7. (Color online) Data monitoring system of the rotor.

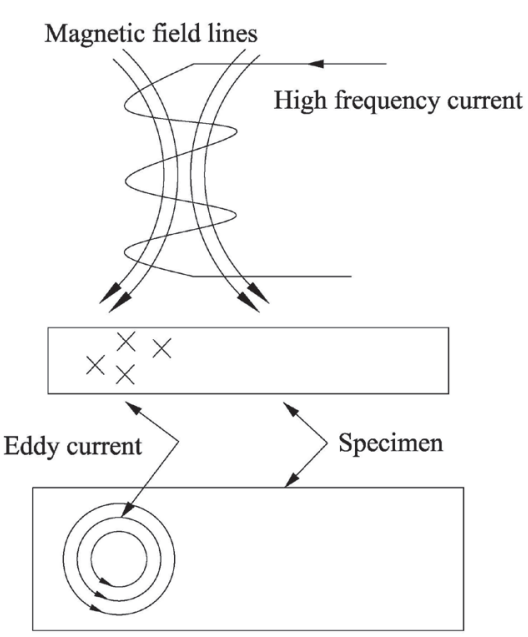

Fig. 8. Operation principle of the eddy current sensor. 


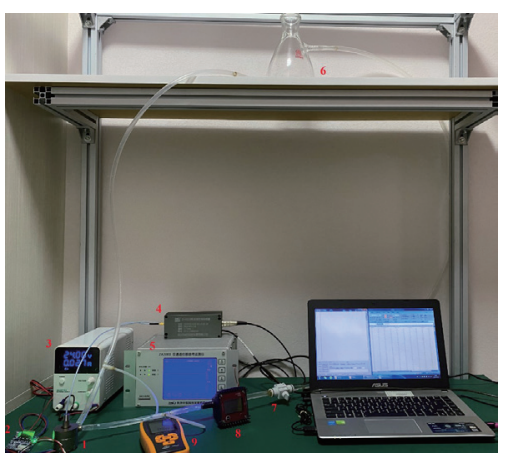

(a)

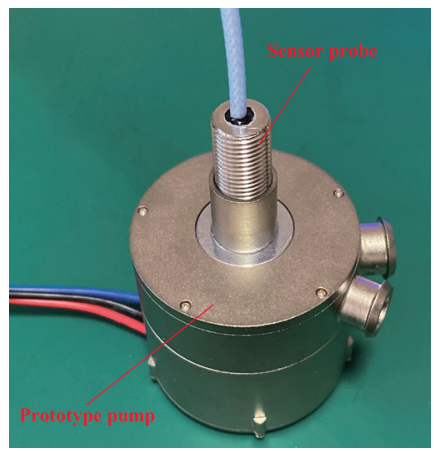

(b)

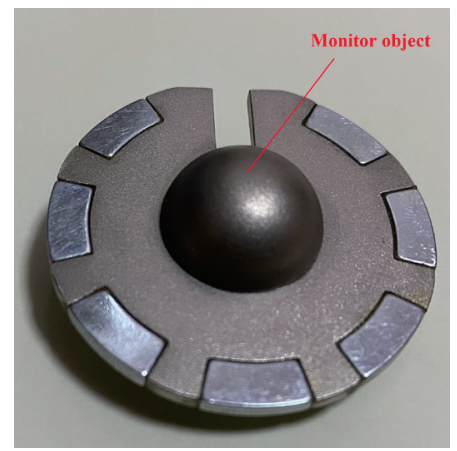

(c)

Fig. 9. (Color online) (a) Test platform for the levitation state of the rotor. 1: Prototype pump. 2: Control device. 3: Power supply system. 4: Non-contact eddy current sensor. 5: Signal monitor. 6: Storage container. 7: Throttle valve. 8: Flowmeter. 9: Differential pressure gauge. (b) Installation of the sensor probe on the prototype pump. (c) Monitored object on the rotor.

storage container, flowmeters, and differential pressure gauge sensors. The real-time levitation state of the rotor was monitored at different speeds using 33\% glycerin solution diluted with saline water as a liquid.

\section{Results and Discussion}

To confirm the principle of our newly developed magnetic levitation blood pump and the mathematical model of the levitation stability for its magnetic bearing, a hydraulic experimental measurement with various sensing components and a levitation state monitoring test were carried out. The measured pressure head versus the outflow rate is shown in Fig. 10. This resulting curve shows the performance of the pump. A continuous output flow rate of $5 \mathrm{~L} / \mathrm{min}$ was obtained against a $100 \mathrm{mmHg}$ pressure head when the rotational speed was approximately $1600 \mathrm{rpm}$. In addition, for a motor speed of $1600 \mathrm{rpm}$, the pressure difference between the inlet and outlet increased from 95 to $115 \mathrm{mmHg}$, while the output flow rate decreased by only about $0.5 \mathrm{~L} / \mathrm{min}$. This test result suggests that the output flow of this pump is stable at different pressure heads.

Figure 11 shows the levitated states of the rotor in cases with and without magnetic rings in the bearing. It can be seen that, in the case without a magnetic ring, the suspension gap of the rotor is approximately zero when the rotational speed and the pressure head are kept at $1700 \mathrm{rpm}$ and $100 \mathrm{mmHg}$, respectively. However, under the same experimental conditions, the suspension gap of the rotor in the case without a magnetic ring is nearly $0.33 \mathrm{~mm}$. It is clear that the rotor of the pump can be levitated by the passive magnetic bearing. To further explore the levitation stability of the rotor, the suspension gap of the rotor at different rotational speeds was monitored. Figure 12 shows the real-time test result of the rotor's levitated state. The average suspension gaps were $0.301,0.365$, and $0.32 \mathrm{~mm}$ when the rotational speeds of the rotor were 1300, 1800, and $2300 \mathrm{rpm}$, respectively. However, the levitated state of the rotor was found to be unstable at low rotational speeds. The suspension gap of the rotor fluctuated between 0.142 and $0.65 \mathrm{~mm}$ at the rotational speed of $1300 \mathrm{rpm}$. With increasing rotational speed, the levitated states of the 


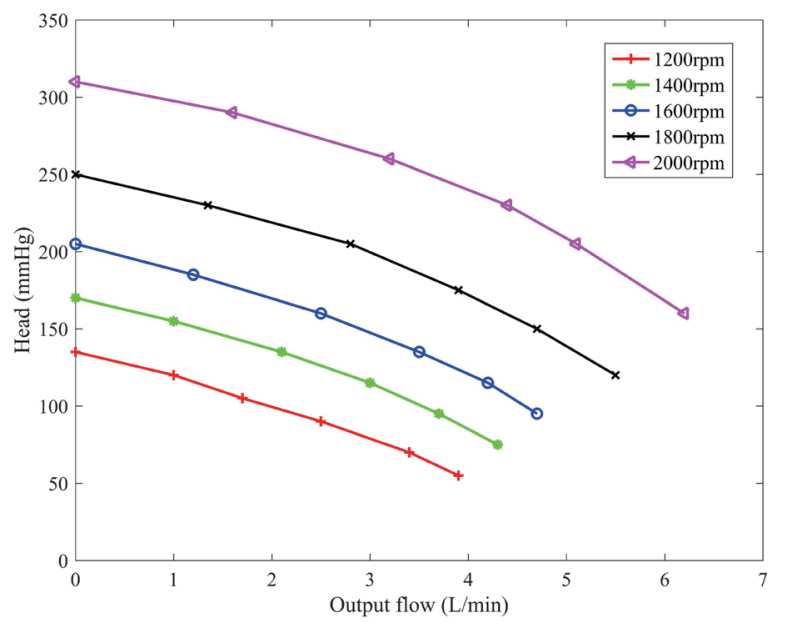

Fig. 10. (Color online) Hydraulic performance of our newly developed blood pump.

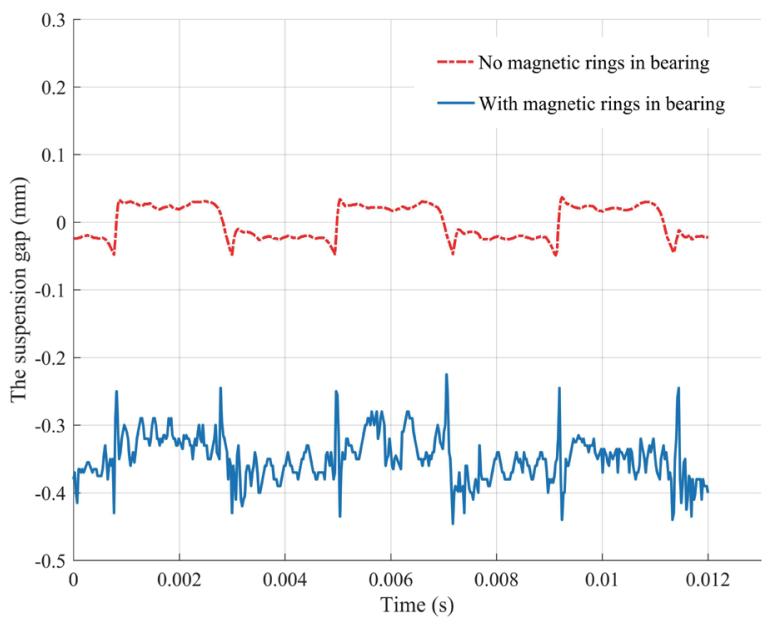

Fig. 11. (Color online) Suspension gap of rotor with and without magnetic rings in the bearing.

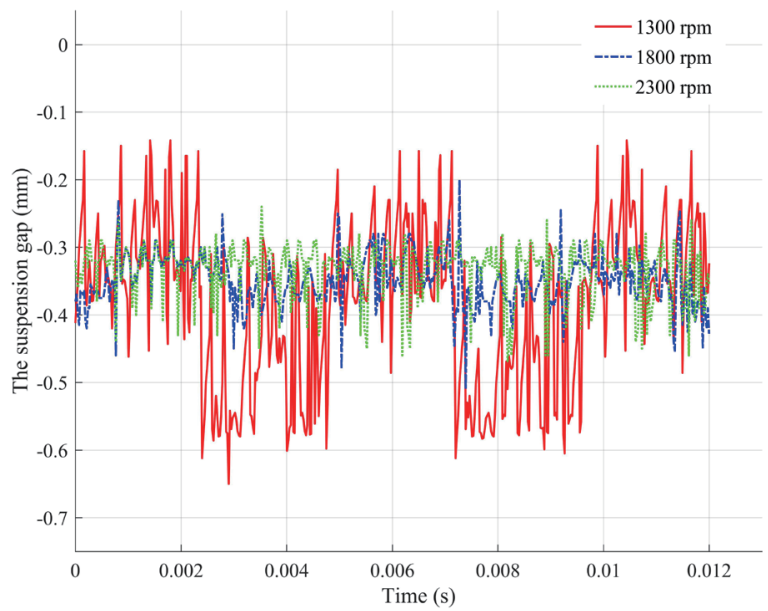

Fig. 12. (Color online) Comparison of suspension gap at various rotational speeds. 
rotor became more stable. The maximum fluctuation of the suspension gap was found to be only $0.2 \mathrm{~mm}$ at a rotational speed of $2300 \mathrm{rpm}$.

In this study, we proposed some valuable principles of the nutation blood pump for constructing a next-generation VAD. We also overcame some difficulties in developing a practical pump. We confirmed the basic principles for the operation of the nutation blood pump proposed in Ref. 7. In addition, we developed more comprehensive models and performed some real-time test measurements toward solving the problems of levitation stability in a nutation blood pump.

\section{Conclusion}

A novel passive magnetically levitated nutation pump was proposed in this paper. By focusing on the levitation stability of the nutation rotor in the static state, a mathematical model was derived to analyze the rotor system's axial stiffness and rotational stiffness based on the theory of equivalent magnetic charges. According to our theoretical study, the magnetic bearing can be levitated stably under the application of axial external forces; however, it cannot remain stable under the action of external torques.

In consideration of the dynamic stability, we introduced the gyro effect to enhance the levitation stability and further derive an analytical expression for the gyroscopic moment of the nutating rotor. Moreover, to explore the dynamic stability of the levitated rotor in our new design pump, a real-time monitoring test measurement with various sensing components was performed. It was found that the rotor can achieve stable levitation. To test the effect of small fluctuations on the suspension gap, the rotational speed of the rotor in the experiment should be as high as possible. In addition, it was found that a continuous output flow rate of $5 \mathrm{~L} / \mathrm{min}$ can be obtained against a pressure head of $100 \mathrm{mmHg}$ and that the output flow conditions of this pump are stable at different pressures. The satisfactory hydraulic performance and stable dynamic levitation validated the design concept of the proposed magnetic levitated nutation pump. The study provides a foundation for the future design of a magnetic levitation ventricular assist nutation pump.

\section{Acknowledgments}

This research was supported by the Natural Science Foundation of Fujian Province (grant number 2019J01822) and the National Natural Science Foundation of China (grant number 51775114).

\section{References}

1 H. Wataru, M. Takuro, M. Tomotaka, S. Daisuke, and M. Osamu: Artif. Organs 44 (2020) 594. https://doi. org/10.1111/aor.13632

2 M. Toru: Proc. Inst. Mech. Eng. Part I: J. Syst. Control Eng. 231 (2016) 330. https://doi. org/10.1177/0959651816640681

3 E. Okamoto, Y. Ishida, T. Yano, and Y. Mitamura: J. Artif. Organs 18 (2015) 181. https://doi.org/10.1007/ $\underline{\text { s10047-014-0806-9 }}$ 
4 E. Okamoto, T. Yano, Y. Shiraishi, and H. Miuea: Artif. Organs 39 (2015) 701. https://doi.org/10.1111/aor.12558

5 P. Jain, D. Robson, S. Shehab, K. Muthiah, P. Jansz, M. R. Qiu, W. Barrett, V. Sivasubramaniam, N. Kumaradevan, P. S. Macdonld, and C. S. Hayward: Cardiovasc. Pathol. 44 (2020) 107156. https://doi. org/10.1016/j.carpath.2019.107156

6 M. Viganò, F. Gazzoli, R. Hetzer, D. Saeed, L. A. R. Golding, M. Morshius, A. Alloni, R. Koerfer, A. ElBanayosy, and L. Arusoglu: Artif. Organs 37 (2013) 121. https://doi.org/10.1111/j.1525-1594.2012.01539.x

7 G. Chen, L. G. Yao, R. Y. Zheng, L. Zhang, and J. X. Ding: IEEE Access 7 (2019) 169327. https://doi. org/10.1109/ACCESS.2019.2955027

8 T. Soni, J. K. Dutt, and A. S. Das: IEEE Trans. Ind. Electron. 67 (2020) 1160. https://doi.org/10.1109/ TIE.2019.2898604

9 C. Jarroux, J. Mahfoud, B. Defoy, and T. Alban: ASME J. Vib. Acoust. 142 (2020) 031004. https://doi. org/10.1115/1.4046124

10 J. P. Yu, Y. Zhou, H. Y. Zuo, K. Z. Zhang, P. F. Liu, Y. B. Li, P. C. Pu, L. Zhao, and Z. Sun: Appl. Comput. Electromagn. Soc. J. 34 (2019) 547.

11 G. Zhang, Q. T. Meng, Y. Y. Zhong, J. Zhang, H. L. Zhang, and M. Fan: J. Mech. Eng. 51 (2015) 56. https://doi. org/10.3901/JME.2015.05.056

12 C. F. Xia, Y. W. Cai, and Y. Ren: Proc. Inst. Mech. Eng. Part I: J. Syst. Control Eng. 233 (2018) 548. https://doi. org $/ 10.1177 / 0959651818800913$

13 P. Ambwani, P. Xu, G. Haugstad, J. S. Jeong, R. Deng, K. A. Mkhoyan, B. Jalan, and C. Leighton: IEEE Trans. Ind. Appl. 54 (2018) 1264. https://doi.org/10.1109/TIA.2017.2766038

14 S. Shida, T. Masuzawa, M. Osa, and R. Sato: Adv. Biomed. Eng. 7 (2018) 63. https://doi.org/10.14326/abe.7.63

15 T. Bente, P. Mathieu, G. Marcus, S. D. Marianne, K. Rolf, N. Antonia, and M. Mirko: Artif. Organs 42 (2018) 510. https://doi.org/10.1111/aor.13074

16 Y. Zhang, Y. Leng, J. Liu, and D. Tan: J. Magn. 24 (2019) 392. https://doi.org/10.4283/JMAG.2019.24.3.392

17 J. G. Li, Q. C. Tan, and Y. C. Pei: Int. J. Appl. Electromagn. Mech. 49 (2015) 169. https://doi.org/10.3233/jae$\underline{150021}$

18 M. Michaelis, B. Bingham, M. Charlton, and C. A. Isaac: Eur. J. Phys. 42 (2020) 015001. https://doi. org/10.1088/1361-6404/abbc2c

19 M. M. Michaelis and D. B. Taylor: Eur. J. Phys. 36 (2015) 065003. https://doi.org/10.1088/01430807/36/6/065003

20 B. Elvio and D. Cristiana: Meccanica 51 (2015) 763. https://doi.org/10.1007/s11012-015-0238-5

21 Y. Z. Liu: Gyro Mechanics (Science Press, Beijing, 2009) 1st ed., pp. 33-35. 